\title{
Solitary large lunate osteochondroma affecting wrist motion: case report
}

\begin{abstract}
Osteochondromas (OC) affecting long bones in children is a well-recognized entity, but isolated osteochondromas involving the carpal bones seem to present a lower incidence and severity. We would like to present a case of a teenager boy with no symptoms of lump formation or deformity, who debuted with a clinic of a severe restriction of wrist motion, due to a rather large lunate tumour invading the midcarpal joint, the radiocarpal joint and up to the TFCC area. The presentation of this pathology affecting the Lunate bone is extremely rare and has not been previously reported.
\end{abstract}

Volume 6 Issue I - 2016

\author{
L Heras Garcia \\ Advanced Surgery, Burjeel Hospital, United Arab Emirates
}

Correspondence: L Heras Garcia, Department of Hand Surgery, Burjeel Hospital for Advanced Surgery, Dubai, United Arab Emirates, Tel (971)566191616, Email l.herasg@gmail.com

Received: October 07, 2016 | Published: October 08, 2016

\section{Introduction}

Osteochondromas are overgrowing or bone projection covered by cartilage which appears most commonly in the metaphysis of long bones, but also have been reported on the diaphysis, in flat bones and in vertebrae, ${ }^{1}$ grow during childhood through adolescence, but usually growing ends when the epiphyseal plates close.If the growth happen in adulthood suggests the diagnosis of malignant transformation to a chondrosarcoma, this transformation may occur in less than $1 \%$ and a maximum of $25 \%$ of cases. ${ }^{2}$

The multiple hereditary osteochondromata is transmitted as autosomal dominant, ${ }^{1}$ but the isolated $\mathrm{OC}$ is rarer and seems to be mainly due to recessive transmission.

The occurrence of an isolatedOC in the carpus is very rare and its excision is indicated in the case of significant symptoms or change in

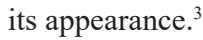

\section{Case report}

A 14 year old boy came to the consultation room of our hospital complaining of pain and limited motion of his right wrist for more than three years, referring a gradually deterioration of his wrist, recently even associated to a continuous pain, the situation became so extreme that he was unable to write anything without discomfort and the gradually reduction of motion was interfering with his daily living activities. In general the function of his right dominant wrist was severely deteriorated.

On examination, he presented a clear reduction of range of motion compared to his left wrist, being able only to extend it up to 50 degree (Figure 1A) and the flexion was more restricted only being able to go to 30 degrees (Figure 1B), leaving an arch of motion of only 50 degrees, his radial deviation was not restricted, but his ulnar deviation was only of 20 degrees (Figure 1C), being actually a very elastic boy, made those reduction of motion even more prominent.

For accurate diagnosis having only a conventional X-ray examination was not adequate, $\mathrm{CT}$ scan and even a contrast MRI test should be performed.

The X-rays showed the presence of a large bone tumour arisen from the lunate bone, not showing the actual involvement of the tumour in the carpal joints (Figure 2A); the subsequent CT scan confirmed the more accurate extension of the mass showing how the osteochondroma expanded dorsally and proximally, reducing and invading the ulno-carpal space (Figure 2B \&2C), and also in the lateral view was easy to see how the deformed lunate had an expansion of the volar rim up to the neck of the capitate, reducing the midcarpal motion as well (Figure 2D).

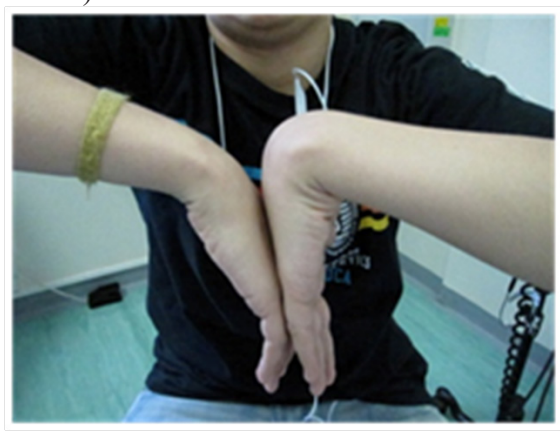

Figure IA Flexion right wrist compare to left side.

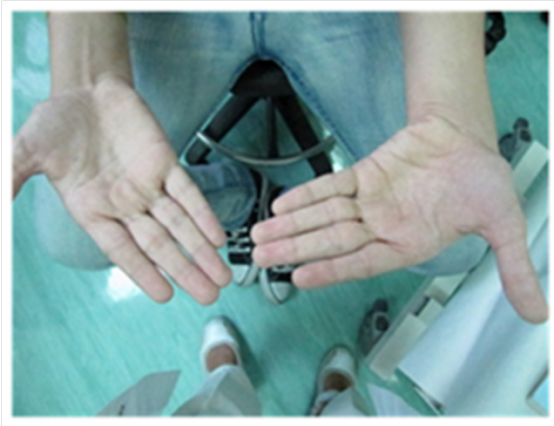

Figure IB Extension right wrist compare to left side.

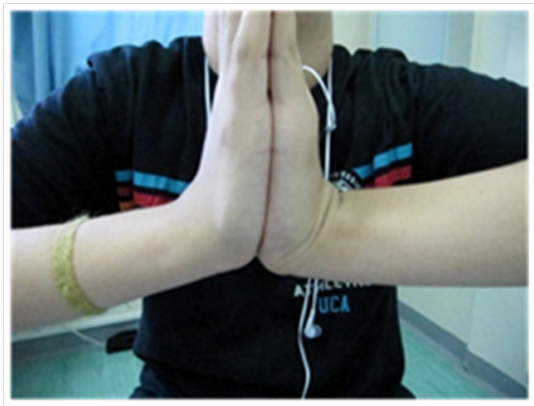

Figure IC Ulnar deviation comparing right with left. 


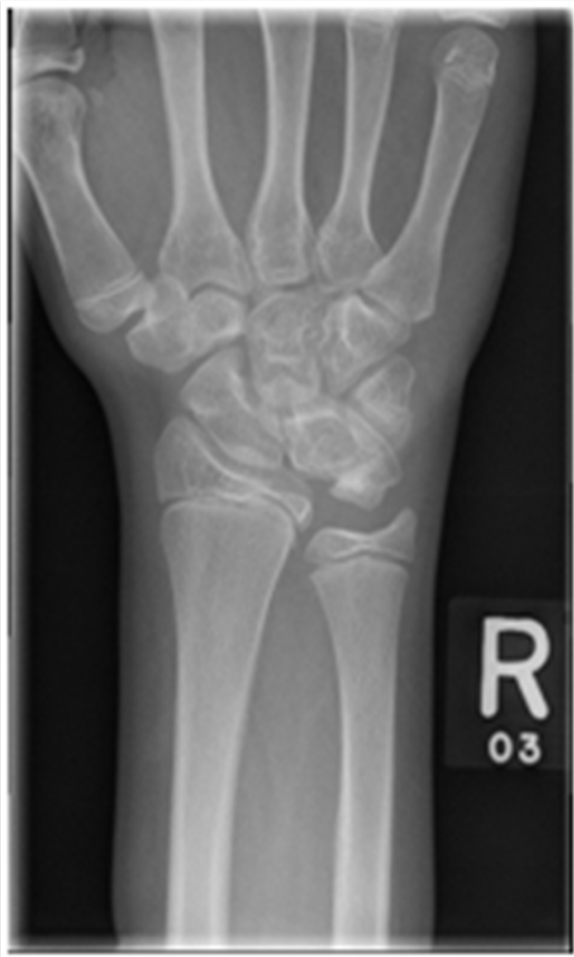

Figure 2A PA x-ray showing the osteochondroma of the ulna.
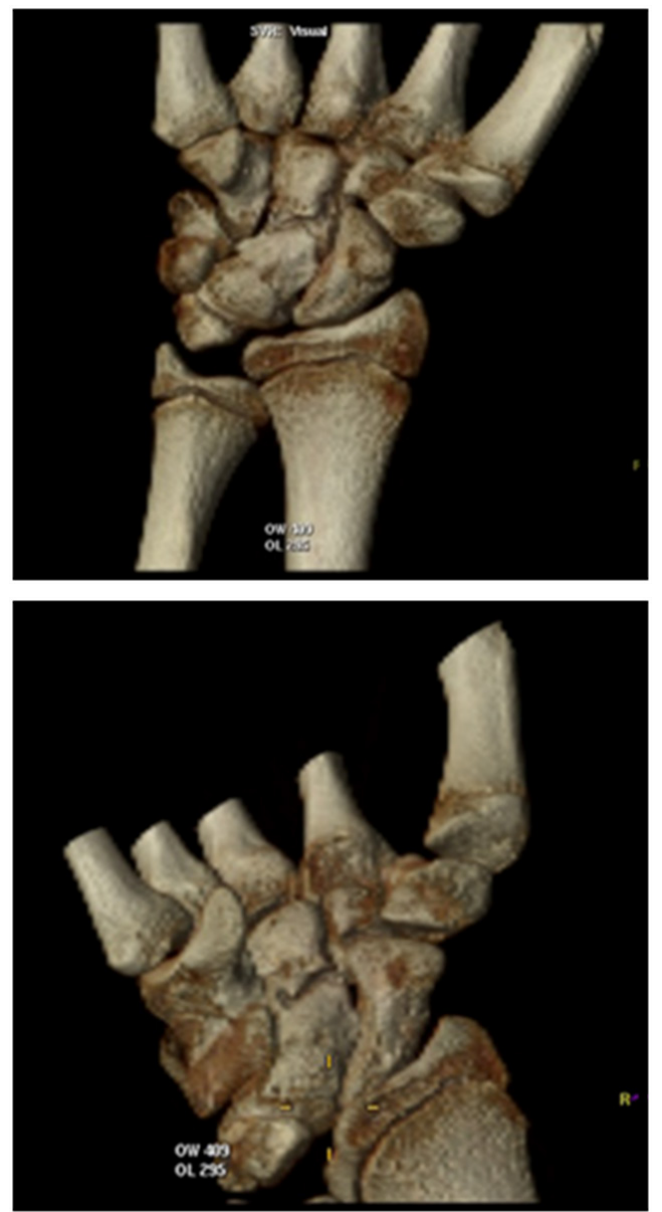

Figure 2B \& C 3D CT scan of the Lunate osteochondroma

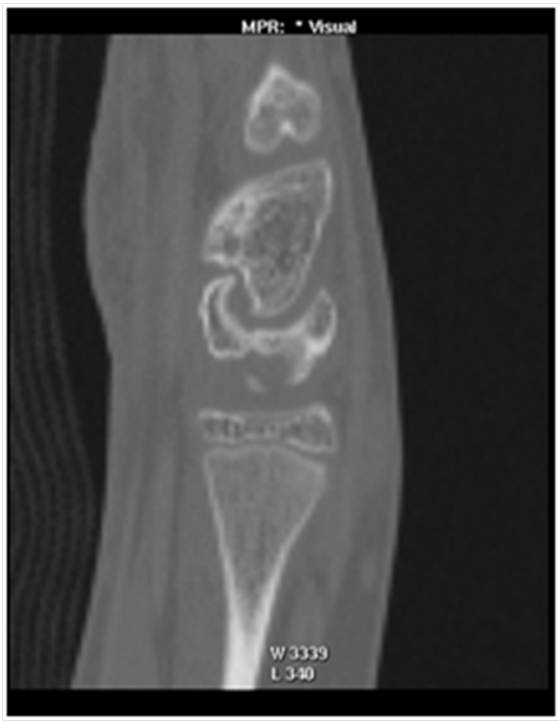

Figure 2D Lateral view showing the extension of the volar rim of the Lunate up to the neck of the Capitate.

The surgery was done later on under general anaesthesia and with tourniquet control, the right wrist was approached dorsally with a longitudinal incision over the $4 / 5$ extensor compartment, the capsule was opened with a ligament sparing technique, and a very large cartilaginous bone tumour, suggesting a osteochondroma, was found extended from dorsal to volar and to the ulnar side, sitting the main mass distal to the triangular fibrocartilaginous complex, where appeared to grow in a more round, smooth shape (Figure 3A-3D).

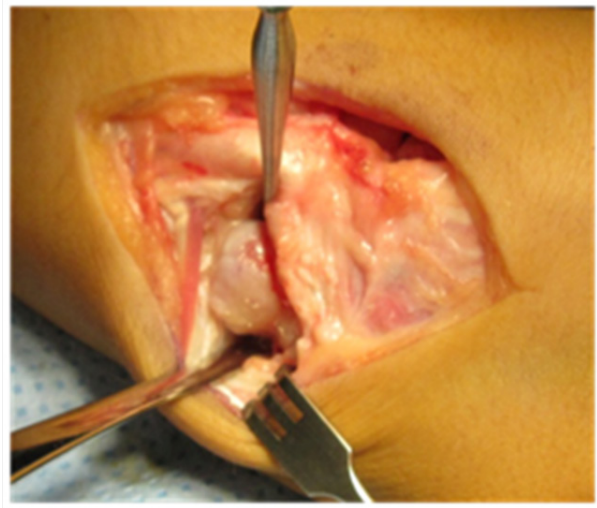

Figure 3A Ulnar side of the osteochondroma.

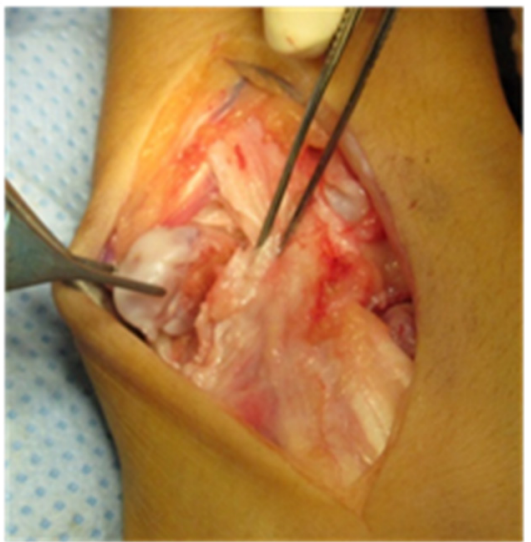

Figure 3B Removal of ulnar extension of the osteochondroma. 


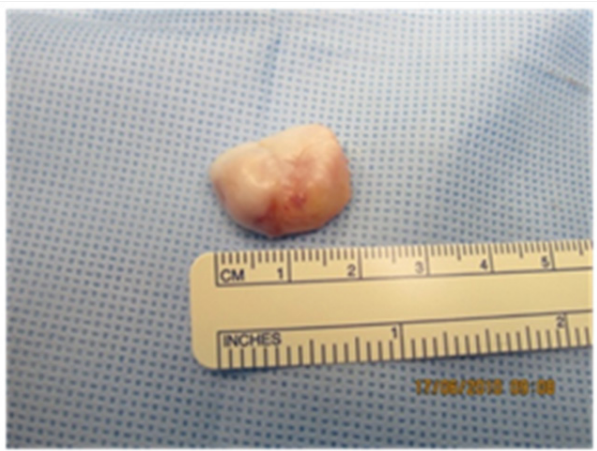

Figure 3C Piece showing the cartilaginous cover typical of the ostechondroma.

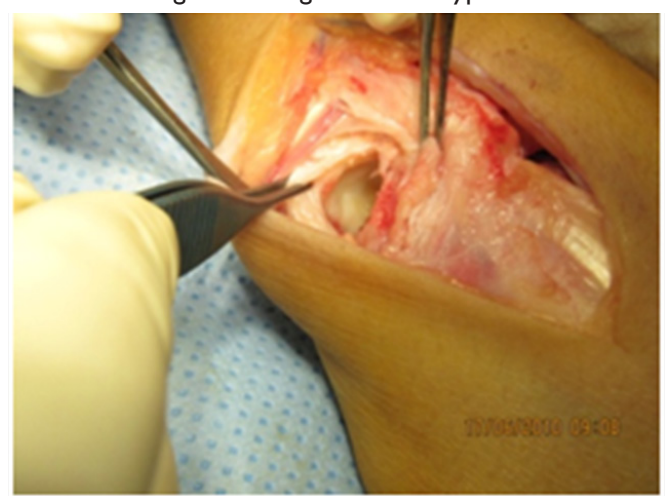

Figure 3D Seen clearly the distal portion of the TFCC undamaged.

A large tumour was removed measuring $2.0 \mathrm{~cm}$ for $1.5 \mathrm{~cm}$ and for $1.0 \mathrm{~cm}$, and a second smaller one was also respected from the volar aspect of the lunate, trimming the fragment that was blocking the lunocapitate joint, that restoredthe ulnocarpal space distal to the TFCC and at the same time improved the wrist motion at the midcarpal joint.

Both specimens were sent to histology for final diagnosis. Histology is needed to confirm the benign tumour, malignancy is rare, but cases have been reported. Pathology report confirmed the presence of a solitary benign large $\mathrm{OC}$, without sign of malignancy.

He had an uneventful recovery from the surgery and the rehabilitation program started one week post-surgery with a hand physiotherapist. Full range of motion was achieved 18 weeks after the intervention being able to write again and return to his previous sport activities. No evidence of recurrence was detected after 6 years.

\section{Discussion}

Hereditary multiple exostoses (HME) is an autosomal dominant inheritable disorder of enchondral bone growth; the cartilaginous exostoses, also called osteochondroma grow from the physes of long bones, pelvis, ribs, scapula and vertebrae. ${ }^{1}$ The prevalence is approximately 1 in $50.000,{ }^{4}$ more severe in males and half of all cases present an involvement of the forearm.

In the English and German medical literature have been reported 327 solitary osteochondromata of the hand, of those there were only four located in the carpus, 70 in the metacarpals and 283 in the phalanges. ${ }^{5}$

Every report confirm that $\mathrm{OC}$ formation in carpal bones are a more unusual entity, reported in the literature as case reports or short reports of three to five cases. The more involved carpal bone is the scaphoid, ${ }^{6,7}$ of which have been reported about twenty cases in total, some the OC presented themselves with just episodes of pain. ${ }^{8}$

But may debut producing Median Nerve compression, ${ }^{9}$ tendon attrition and rupture, ${ }^{10}$ and have been reported rare cases of carpal scapho-lunate dissociation ${ }^{11}$ or mimicking carpal coalition. ${ }^{12}$

In even rarer cases the exostoses may affect the Capitate or the Hamate bones, having only three papers in the literature where were described the involvement of those bones, ${ }^{3,13,14}$ in two of those cases arose a possible diagnoses of osteosarcoma which proved otherwise with the histology

The case presented in this paper is a rather large OC affecting the Lunate bone which is extremely rare, only another case has been reported ${ }^{15}$ with a formation of a small OC producing a snapping on the Median Nerve.

Osteochondromas in the carpal bones tends to be of small size, possible due to the lack of space, that prevents the expansion around the Scaphoid or the Capitate bones, but the situation is different for the Lunate, the partial articulation of this bone with the TFCC leaves plenty of space at the level of the ulno-carpal space for the OC to continue growing and may develop like in this case a much larger tumour which will fill the whole ulno-carpal space blocking the wrist motion, this does not occur in the OC involving the scaphoid, capitate or trapezium.

The smaller size of most of the carpal OC preclude the surgical intervention, but tumour of larger size, and those intra-articular and transosseous lesions that are more likely to result in angular deformities and loss of motion at the joints should be recommended to undergoes an early surgical removal to regain the wrist function and to confirm that there is no sign of malignancy in the histology report.

\section{In resume}

Osteochondroma is the most benign tumour of the bone. In the hands more typically originates from the metaphysis of the long bones, metacarpal and phalange as, being rare seen them affecting the carpal bones. Tends to appears frequently in the second decade and has a predominance of 1.7 to 1.0 .

The indications for surgical treatment are pain, functional limitation and cosmetic reasons. An aggressive intervention is necessary when deformity or sever limitation of activity of daily living are present.

Malignant degeneration is very rare in isolate presentations, but can be seen in 10 to $20 \%$ of patients affected of multiple osteochondromas. $^{3}$

\section{Acknowledgments}

None.

\section{Conflicts of interest}

None.

\section{References}

1. Peterson HA. Multiple hereditary osteochondromata. Clin Orthop Relat Res. 1989;239:222-230.

2. Sansón RíoFrío JA, Santiesteban N, Bahena RI, et al. Differential diagnosis of multiple hereditary exostosis: presentation of a clinical case with secondary chondrosarcoma and literature review. Acta Ortop Mex. 2009;23(6):376-382. 
3. Roulot E, Malikov S, Green JA, et al. Osteogenic exostosis of the capitate bone. Case report and review of the literature. Skeletal Radiol. 2003;32(3):121-127.

4. Schmale GA, Conrad EU, Raskind WH. The natural history of hereditary multiple exostoses. J Bone Joint Surg Am. 1994;76(7):986-992.

5. Gaulke R. The distribution of solitary enchondromata at the hand. $J$ Hand Surg Br. 2002;27(5):444-445.

6. Harris NJ, Bell MJ. Bilateral scaphoid exostoses. J Hand Surg Br. $1995 ; 20(6): 745$.

7. Van Alphen JC, Te Slaa RL, Eulderink F, et al. Solitary osteochondroma of the scaphoid: a case report. Journal of Hand Surgery. 1996;21(3):423-425.

8. Spinner RJ, Spinner M. Superficial radial nerve compression due to a scaphoid exostosis. J Hand Surg Br. 1996;21(6):781-782.

9. Hofmann AK, Wüstner MC, Spier W. Compression neuropathy of the median nerve at the wrist joint caused by chondroma. Handchir Mikrochir Plast Chir. 1990;22(2):96-98.
10. O Dwyer KJ, Jefferiss CD. Scaphoid exostosis causing rupture of the flexor pollicis longus. Acta Orthopaedica Belgica. 1994;60(1):124-126.

11. De Smet L, Degreef I. Bilateral osteochondroma of the scaphoid causing scapholunate dissociation: a case report. Chir Main. 2007;26(3):141-142.

12. Rice J, Stephens M, Colville J. Scaphoid osteochondroma mimicking carpal coalition. J Hand Surg Br. 1996;21(6):779-780.

13. Bellemère $P$, Chaise F, Friol JP, et al. Solitary carpal osteochondroma Apropos of a case. Ann Chir Main Memb Super. 1994;13(3):179-183.

14. Koti M, Honakeri SP, Thomas A. A multilobed osteochondroma of the hamate: case report. J Hand Surg Am. 2009;34(8):1515-1517.

15. Takagi T, Matsumura T, Shiraishi T. Lunate osteochondroma: a case report. J Hand Surg Am. 2005;30(4):693-695. 